\title{
Semi-analytical theory of motion for the PSR B1257+12 planetary system
}

\author{
Maciej Konacki, Andrzej J. Maciejewski \\ Torun Centre for Astronomy, Nicolaus Copernicus University, \\ 87-100 Toruń, Gagarina 11, Poland \\ Alex Wolszczan \\ Pennsylvania State University, Department of Astronomy and \\ Astrophysics, 525 Davey Lab, University Park, PA 16802, USA \\ Toruń Centre for Astronomy, Nicolaus Copernicus University \\ ul. Gagarina 11, 87-100 Toruń, Poland
}

\section{Introduction}

A planetary system around the pulsar B1257+12 has three planets A, B and C with the orbital periods of 25, 66 and 98 days, respectively (Wolszczan 1994). Dynamical properties of the system have been thoroughly studied by Rasio et al. (1993) and Malhotra (1993). They demonstrated that the gravitational interactions between planets $\mathrm{B}$ and $\mathrm{C}$ are significant enough to be detected. In such case, the motion of the system is no longer Keplerian and it is necessary to use a more precise description of motion in order to model the data properly. In this paper we derive a semi-analytical theory of motion assuming that the relative inclination of the orbits is small. We perform numerical simulations to show that our theory sucessfully predicts times of arrival of pulsar pulses and allows a determination of orbital inclinations and hence the masses of planets $B$ and $\mathrm{C}$.

\section{Theory of motion}

Although the interactions between the orbits of the two planets are no longer Keplerian, we can still use Keplerian description of their motion in terms of the osculating orbital elements (i.e. the elements that change in time). In the case of the PSR B1257+12 planetary system the most significant part of the interactions comes from planets B and C. Thus, a low-mass planet A can be neglected, while analyzing the non-Keplerian part of the motion. Using Hamilton's description of motion, the Hamiltonian $H$ of the system can be represented as the sum of two terms

$$
H=H_{K}+H_{P}
$$

where $H_{K}$ is responsible for the Keplerian motion and $H_{P}$ accounts for the perturbations (i.e. the change of otherwise constant orbital elements). The perturbative part of Hamiltonian expanded to the first order in relative inclination of the orbits and the product of masses of planets $\mathrm{B}$ and $\mathrm{C}, m_{1} m_{2}$, has the 


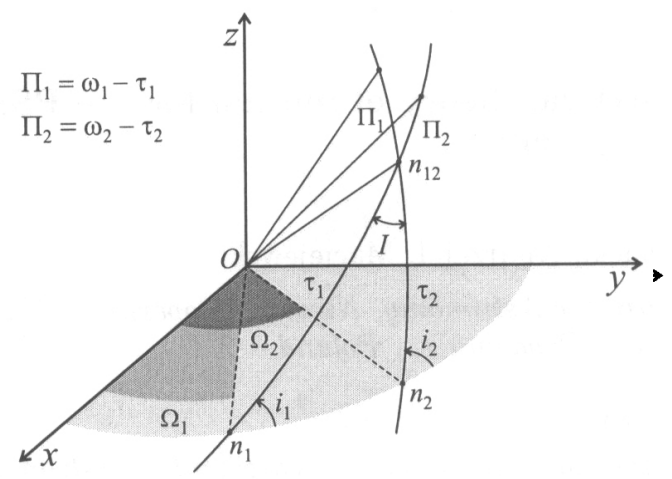

Figure 1. Geometry of the system: $\tau_{1}$ and $\tau_{2}$ are the angles $n_{1} O n_{12}$ and $n_{2} O n_{12}$ respectively. The angles $I$ (relative inclination of the orbits), $\tau_{1}$ and $\tau_{2}$ can be found by solving the spherical triangle $n_{1} n_{12} n_{2}$.

following form

$$
H_{P}=-\frac{G m_{1} m_{2}}{r_{2}}\left[\left(1-2 \frac{r_{1}}{r_{2}} \cos \psi+\left(\frac{r_{1}}{r_{2}}\right)^{2}\right)^{-1 / 2}-\frac{r_{1}}{r_{2}} \cos \psi\right]
$$

where

$$
\psi=f_{1}+\omega_{1}-f_{2}-\omega_{2}-\tau, \quad \tau=\tau_{1}-\tau_{2}, \quad r_{j}=\frac{a_{j}\left(1-e_{j}^{2}\right)}{1+e_{j} \cos f_{j}}, \quad j=1,2,
$$

$f_{j}$ is the true anomaly, $a_{j}$ is the semi-major axis, $e_{j}$ is the eccentricity and $\omega_{j}$ denotes the argument of pericenter. The parameter $\tau$ is a function of orbital inclinations $i_{j}$ and longitudes of ascending node $\Omega_{j}$ (" 1 " corresponds to planet $\mathrm{B}$ and "2" to planet $\mathrm{C}$, see Fig. 1). The assumption that a relative inclination of the orbits of planets $\mathrm{B}$ and $\mathrm{C}$ is small implies that $i_{j}$ and $\Omega_{j}$ are approximately constant. Thus the remaining elements that change in time are $a_{j}, e_{j}, \omega_{j}, T_{p j}$, where $T_{p j}$ denotes the time of pericenter. In fact, instead of $T_{p j}$, it is convenient to use mean longitude $\lambda_{j}=M_{j} t+\sigma_{j}+\omega_{j}$, where $\sigma_{j}=-M_{j} T_{p j}$ and $M_{j}$ is the mean motion. Next, from the classical form of Lagrange's perturbation equations we obtain the following set of the first order differential equations for the elements $a_{j}, e_{j}, \omega_{j}, \lambda_{j}$

$$
\dot{a}_{j}=\frac{-2}{\mu_{j} M_{j} a_{j}} \frac{\partial H_{P}}{\partial \sigma_{j}}
$$




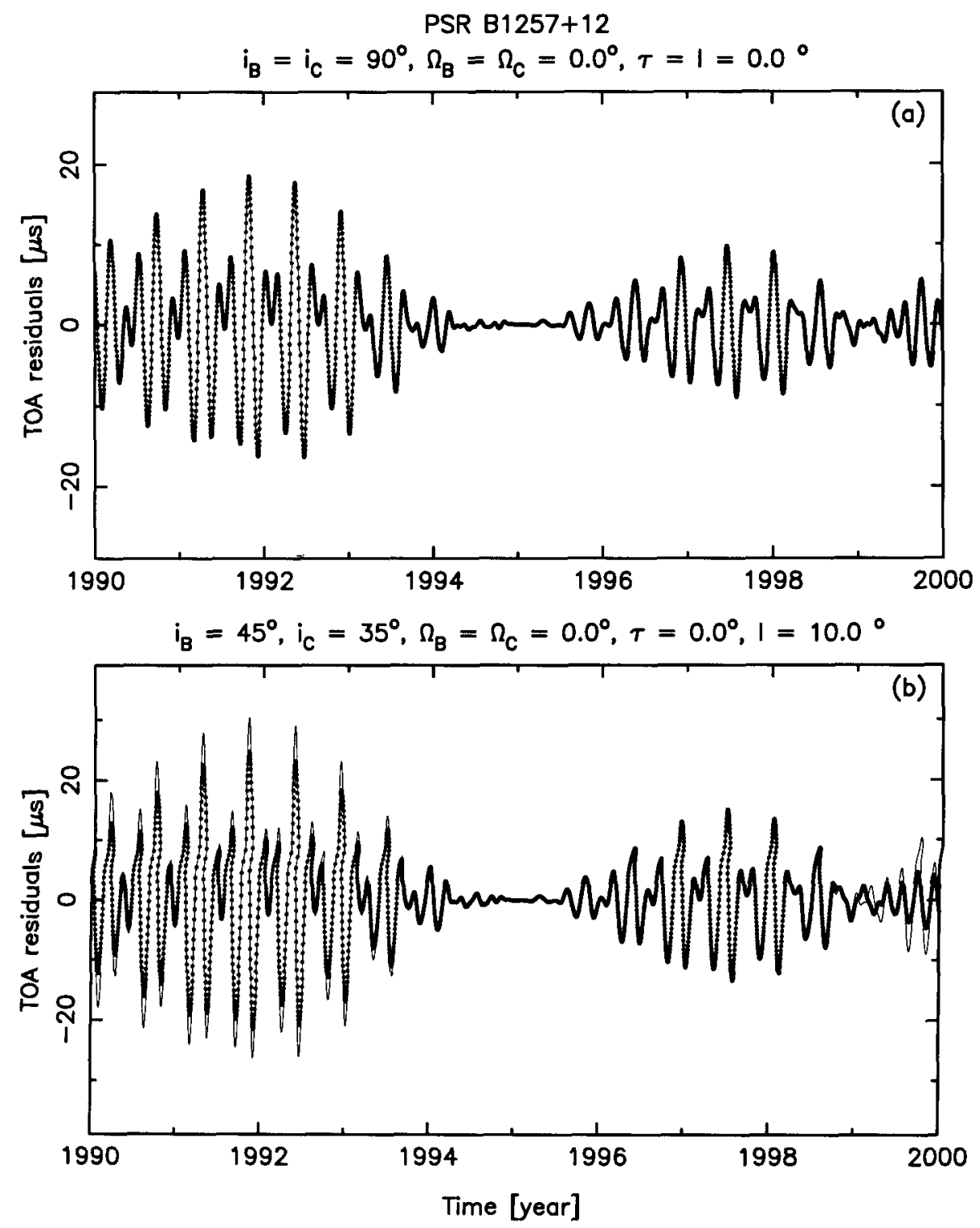

Figure 2. TOA residuals due to the non-keplerian part of motion of PSR B $1257+12$. The solution obtained by means of the numerical integration of full equations of motion is indicated with the dots and the one obtained using our model is marked with the solid line. 


$$
\begin{gathered}
\dot{e}_{j}=\frac{1}{\mu_{j} M_{j} a_{j}^{2} e_{j}}\left[-\left(1-e_{j}^{2}\right) \frac{\partial H_{P}}{\partial \sigma_{j}}+\sqrt{1-e_{j}^{2}} \frac{\partial H_{P}}{\partial \omega_{j}}\right], \\
\dot{\omega}_{j}=\frac{-\sqrt{1-e_{j}^{2}}}{\mu_{j} M_{j} a_{j}^{2} e_{j}} \frac{\partial H_{P}}{\partial e_{j}}, \\
\dot{\lambda}_{j}=M_{j}+\frac{2}{\mu_{j} M_{j} a_{j}} \frac{\partial H_{P}}{\partial a_{j}}-\frac{e_{j} \sqrt{1-e_{j}^{2}}}{\mu_{j} M_{j} a_{j}^{2}\left(1+\sqrt{1-e_{j}^{2}}\right.} \frac{\partial H_{P}}{\partial e_{j}} .
\end{gathered}
$$

Given the above formulae and the form of the perturbing Hamiltonian $H_{P}$, one can easily obtain the explicit form of the right-hand-side functions of the equations for osculating elements. These equations can be solved numerically for $a_{j}, e_{j}, \omega_{j}, \lambda_{j}$. Finally, from the above equations it follows that changes of the orbital parameters of planets $\mathrm{B}$ and $\mathrm{C}$ are proportional to $m_{2} / m_{0}$ and $m_{1} / m_{0}$, respectively, with $m_{0}$ denoting the pulsar mass.

\section{Tests}

The above theory can be applied to make very precise predictions of the times of arrival of pulsar pulses (TOAs). In principle, the variations of TOA residuals due to the orbital motion of the pulsar can be represented as a sum of two terms

$$
\Delta t(t)=\Delta t_{K}(t)+\Delta t_{P}(t)
$$

where $\Delta t_{K}(t)$ denotes the Keplerian part of the motion and $\Delta t_{P}(t)$ is the part due to interactions between planets B and C. In Fig. 2, we compare the behavior of $\Delta t_{P}(t)$ predicted by our model with the results of numerical integration of full equations of motion in two different configurations of the system. Clearly, our model is indistinguishable from the one based on numerical integration for the coplanar orbits and it only slightly diverges from the exact solution, when a relative inclination of the orbits reaches $\sim 10^{\circ}$. It is important to keep in mind that the theory presented here expresses the TOA residual function $\Delta t_{P}(t)$ in terms of the parameters $m_{2} / m_{0}, m_{1} / m_{0}$ and $\tau$. Consequently, having the theory of orbital motion implemented in the timing model for PSR B1257+12, we can determine the values of planetary masses from the least-squares analysis of the real data. A demonstration of this process on simulated data and the details of the theoretical approach described here will be discussed in our forthcoming paper.

\section{References}

Brouwer, D. \& Clemence, G. M. 1961, Methods of Celestial Mechanics, (New York: Academic Press)

Malhotra, R. 1993, ApJ, 407, 266

Rasio, F. A., Nicholson, P. D., Shapiro, S. L. \& Teukolsky, S. A. 1993, in ASP Conf. Ser. 36, Planets Around Pulsars, ed. J.A. Phillips, S.E. Thorsett, \& S.R. Kulkarni (San Francisco: ASP), 107

Wolszczan, A. 1994, Science, 264, 538 